\title{
Population Synthesis and the Diagnostics of High-redshift Galaxies
}

\author{
Alberto Buzzoni \\ Telescopio Nazionale Galileo, A.P. 565, 38700 S/Cruz de La Palma (Tf), Spain \\ and \\ Osservatorio Astronomico di Brera, Milano Italy
}

\begin{abstract}
The effect of redshift on the observation of distant galaxies is briefly discussed emphasizing the possible sources of bias in the interpretation of high- $z$ data. A general energetic criterion to assess physical self-consistency of evolutionary population synthesis models is also proposed, for a more appropriate use of this important tool to investigate distinctive properties of primeval galaxies.
\end{abstract}

Keywords: Galaxies: high-redshift, evolution, stellar content

\section{Introduction}

The recent major advances in the observation of the deep Universe have substantially improved our chance to reach the redshift of galaxy formation. Objects at $z>5$ begin in fact to emerge from HST observations and other deep surveys with the new-generation telescopes (Cohen et al. 2000).

As far as we move to larger distances, however, optical (and infrared) observations probe galaxy spectral energy distribution (SED) at much shorter restframe wavelength, in the ultraviolet domain. If neglected, this change in our spectral vantage point could lead to an important bias in the interpretation of high-redshift data. In this note, we will briefly analyze some aspects of this problem dealing both with the observational side and the appropriate use of theoretical tools such as population synthesis models to match primeval galaxy evolution.

\section{Redshift constraints and galaxy spectral properties}

One important consequence of redshift (besides the first and most obvious effect of moving spectral features to longer wavelength) is that the wavelength interval $\Delta \lambda_{o b s}$ sampled by any fixed set of photometric bands will span a narrower portion of the restframe SED when observing galaxies at increasing $z$, such as $\Delta \lambda_{\text {rest }}=\Delta \lambda_{\text {obs }} /(1+z)$. 


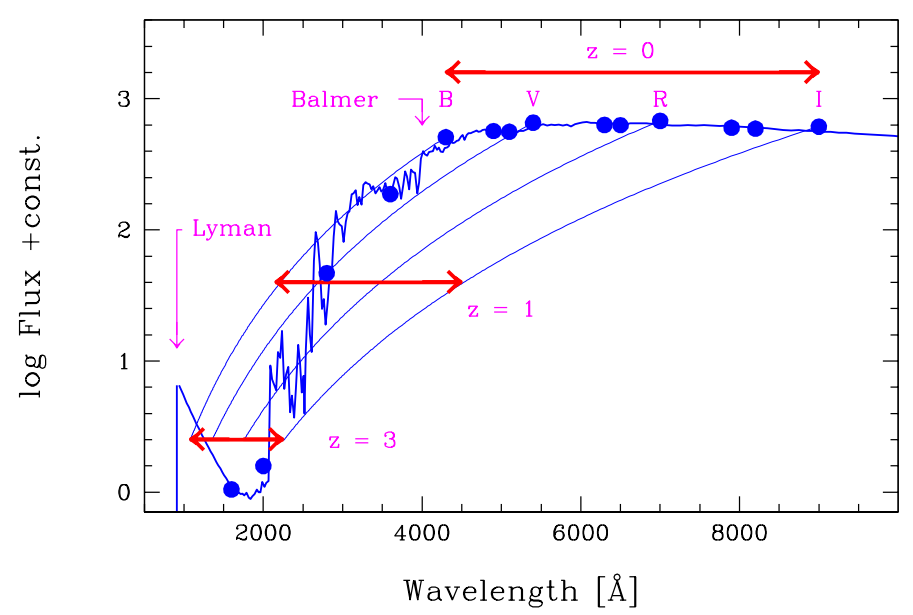

Figure 1. Restframe portion of galaxy SED explored at different redshift by the Johnson four-color photometry through the $B, V, R$, and $I$ bands. The wide wavelength range, between 4000 and $9000 \AA$, attained for local galaxies, "shrinks" to a 1000-2200 $\AA$ interval when observing objects at $z=3$. The synthetic SED of a model elliptical is displayed, for reference.

This effect, by itself, can be of paramount importance as far as we try to gain information on galaxy stellar content by means of multicolor photometry. Figure 1 gives an illuminating example in this sense.

For a $z=0$ galaxy, for instance, Johnson four-color photometry in the $B V R I$ bands would span a wide wavelength range, from 4000 to $9000 \AA$, and collect a substantial contribution from the galaxy stellar population. On the contrary, when observing at $z=3$ with the same photometric system, we would be covering a scarce $\pm 600 \AA$ spectral window centered about $1600 \AA$ in the galaxy restframe. Quite deceivingly, our "multicolor" photometry at $z=0$ would translate into a nearly "monochromatic" estimate of the galaxy flux at $z=3$.

\subsection{Sampling Stellar POPUlations OF High- $z$ Galaxies}

According to the overall color-magnitude distribution, each star in a galaxy aggregate contributes with a different weight to the integrated luminosity with varying wavelength. In Buzzoni (1993) we approached this problem in a statistical way introducing the concept of "effective" contributors in a stellar population. This quantity directly relates to galaxy integrated properties, such as total luminosity or surface brightness fluctuations (as further explored, for instance, by Tonry and Schneider 1988).

We showed, in particular, that by sampling at a given wavelength a total luminosity $L_{\text {tot }}$ provided by $N$ stars, the expected Poissonian 


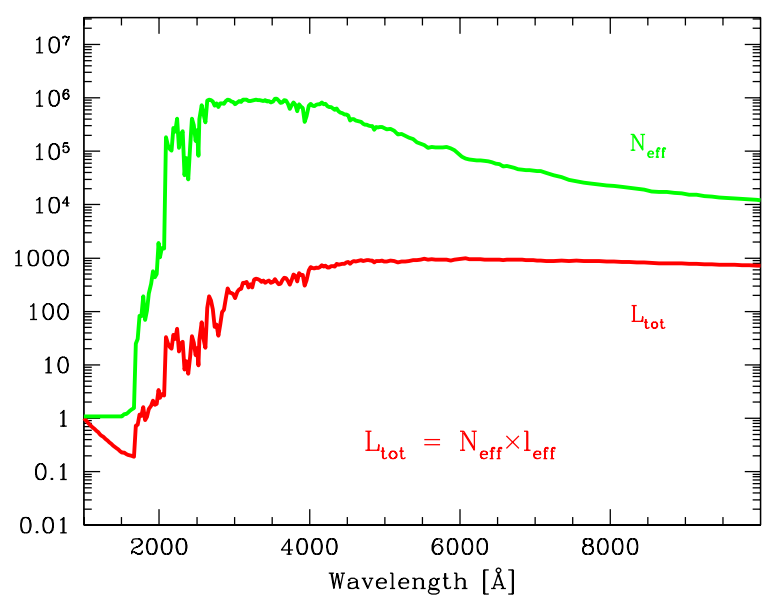

Figure 2. Relative number of "effective" stellar contributors $\left(N_{\text {eff }}\right)$ and SED $\left(L_{t o t}\right)$ for a 5 Gyr SSP model with Salpeter IMF and $[\mathrm{Fe} / \mathrm{H}]=+0.3$ dex according to Buzzoni (1993). Both quantities are in logarithm scale and arbitrarily normalized to their values at $1000 \AA$.

fluctuation results:

$$
\frac{\sigma(L)}{L_{t o t}}=\frac{\left(\sum^{N} l_{i}^{2}\right)^{1 / 2}}{\sum^{N} l_{i}},
$$

where $l_{i}$ is the luminosity of each composing star.

If all stars have the same luminosity, then simply $\sigma(L) / L_{t o t}=$ $1 / \sqrt{N}$. More generally, however, a quantity

$$
N_{\text {eff }}=\frac{\left(\sum^{N} l_{i}\right)^{2}}{\sum^{N} l_{i}^{2}} \leq N
$$

can be defined, as a function of $\lambda$. Its value gives a statistical measure of the effective number of stars that provide $L_{t o t}$ at a given wavelength. Following Buzzoni (1993), a "mean" luminosity $\left(l_{\text {eff }}\right)$ for the composing stars can also be computed as

$$
l_{\text {eff }}=\frac{\sum^{N} l_{i}^{2}}{\sum^{N} l_{i}}
$$

so that, at every wavelength,

$$
L_{t o t}=N_{e f f} \times l_{e f f} .
$$

In Fig. 2 we show the expected value of $N_{\text {eff }}$ for the illustrative case of a 5 Gyr simple stellar population (SSP) with Salpeter IMF and 
$[\mathrm{Fe} / \mathrm{H}]=+0.3$ along with the integrated SED of the model. This should closely resemble the real case of primeval elliptical galaxies (Buzzoni 1995).

As a stricking feature of the model, note that below $2000 \AA$ the effective number of "luminous" contributors dramatically drops by over six (!) orders of magnitude compared for example with the $B$ band value at $4000 \AA$. On the contrary, luminosity over the same wavelength range only dims by a factor of $L_{B} / L_{2000} \sim 10^{3}$. In terms of surface brightness fluctuations this means, for instance, that the ultraviolet image of a galaxy consisting of $10^{11}$ stars should fairly compare with the $B$ observations of a ( $10^{6}$ star-poorer) Galactic globular cluster.

Such a severe undersampling of the galaxy stellar population in the ultraviolet range could have an important impact when studying morphology of $z>1$ ellipticals at optical wavelength (but the same argument holds also for late-type systems). In particular, high-redshift objects would more likely appear as coarser systems with increasing $z$ (e.g. Puerari 2001, this conference).

\subsection{REDShift AND AGE BIAS}

The selective sampling of galaxy stellar population with changing wavelength has even more pervasive consequences, as far as we track evolution of starforming systems at different redshift.

More generally, we could describe a composite stellar population in terms of a suitable convolution of SSPs according to the star formation rate (SFR) at the different epochs. The total luminosity at age " $t$ " results therefore:

$$
L_{t o t}(t)=\int_{0}^{t} L_{S S P}(\tau) S F R(t-\tau) d \tau
$$

A mean luminosity-weighted age of the composing SSPs can also be derived such as

$$
\bar{t}_{*}=\frac{\int_{0}^{t} \tau L_{S S P}(\tau) S F R(t-\tau) d \tau}{L_{t o t}(t)}
$$

This could be regarded as the "representative" age of the stars that contribute to galaxy luminosity. We know (Buzzoni 1995; Tinsley and Gunn 1976) that SSP luminosity evolution can be pretty well described by a simple power law such as $L_{S S P} \propto t^{-\alpha}$ with the power index $\alpha$ that is a function of wavelength. As an instructive example, if we consider for 
the SFR a power-law time decay such as $\mathrm{SFR} \propto t^{-\eta},{ }^{1}$ eq. (6) becomes

$$
\bar{t}_{*}=\frac{\int_{0}^{t} \tau^{1-\alpha}(t-\tau)^{-\eta} d \tau}{\int_{0}^{t} \tau^{-\alpha}(t-\tau)^{-\eta} d \tau} .
$$

The integral has an analytical solution such as

$$
\bar{t}_{*}=\frac{1-\alpha}{2-\alpha-\eta} t
$$

In bolometric, $\alpha=0.76$ (Buzzoni 1989) and, for a constant SFR (i.e. $\eta=0), \bar{t}_{*}=0.2 \mathrm{t}$. At $15 \mathrm{Gyr}$, bright stars are therefore in average 3 Gyr old, but this value changes according to the $\alpha(\lambda)$ function. For a SSP with Salpeter IMF and $Z_{\odot}$, the luminosity power index smoothly increases from $\alpha=0.71$ in the $K$ band to 0.94 in the $B$ band, and exceeds unity below $3500 \AA$. A value of $\alpha \geq 1$ implies that $\bar{t}_{*} \rightarrow 0$, that is only the youngest and more massive stars are representative contributors to galaxy ultraviolet luminosity, as discussed in the previous section.

The mean age of stars contributing to galaxy luminosity at different wavelength for a synthesis model of a 15 Gyr Magellanic irregular (see Buzzoni 2001 for details) is reported in Fig. 3. Note from the figure that the value of $\bar{t}_{*}$ smoothly decreases with decreasing wavelength. As a result, high-redshift objects forcedly appear to be younger than local homologues at $z=0$ in spite of any intrinsic evolution.

\section{Energetic self-consistency of population synthesis models}

Evolutionary population synthesis models are extensively used as a reference tool to reproduce galaxy colors and investigate galaxy spectrophotometric properties at the different cosmic epochs (Buzzoni 1989, 1995, 2001; Bruzual and Charlot 1993; Worthey 1994; Bressan et al. 1994; Fioc and Rocca-Volmerange 1997).

Besides any difference in the computational details and input physics, a crucial constraint that needs to be consistently fulfilled in the synthesis procedure concerns a suitable match of the MS and Post-MS luminosity contribution in the SSP models. The so-called "Fuel consumption theorem" of Renzini and Buzzoni (1986) provides a simple

\footnotetext{
1 The major advantage of this parameterization is that the full range of galaxy morphologies can simply be accounted for by an age-independent distinctive birthrate: $b=\mathrm{SFR} /<\mathrm{SFR}>=(1-\eta)$. This easily derives recalling that, at age $t, \mathrm{SFR}=C t^{-\eta}$ and its time average is $\langle\mathrm{SFR}\rangle=C t^{-1} \int_{0}^{t} \tau^{-\eta} d \tau$. Providing that $\eta<1$, we always have $<\mathrm{SFR}>=\mathrm{SFR} /(1-\eta)$, from which the value of $b$ directly follows, by definition.
} 


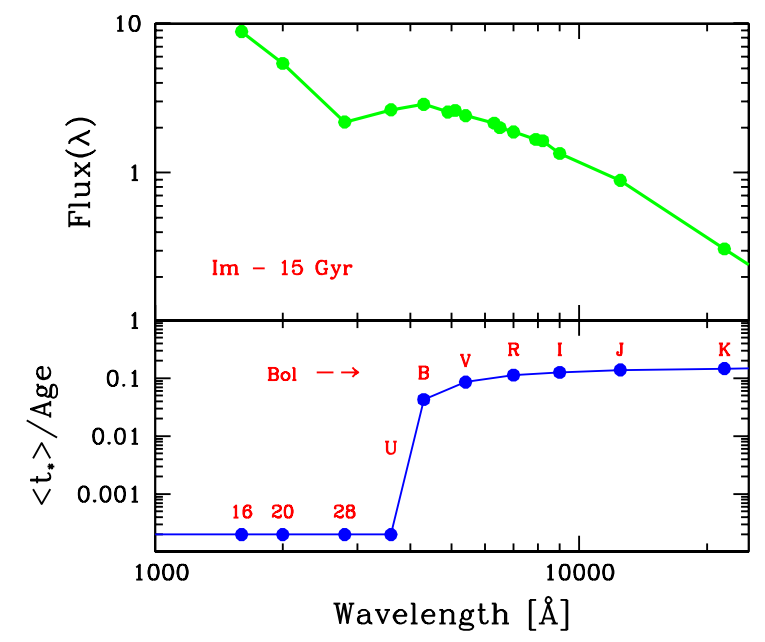

Figure 3. Theoretical SED (upper panel), and "mean" age (relative to galaxy age) of stars at different photometric bands (and in bolometric as well), according to eq. (7) (lower panel), for a 15 Gyr Im model galaxy from Buzzoni (2001).

and very powerful tool in this sense. According to the theorem, the Post-MS bolometric luminosity in a SSP of total luminosity $\left(L_{b o l}\right)$ is

$$
L_{P M S}=\mathcal{B} \times L_{b o l} \times \text { Fuel }
$$

In the equation, the nuclear fuel refers to the amount consumed by one star leaving the MS turn-off (TO) point along the whole Post-MS evolution. This is a natural output of the theoretical stellar tracks and is therefore known with high accuracy. The scaling factor $\mathcal{B}$ is the "specific evolutionary flux" of the SSP, and directly deals with the stellar clock, that is the relationship between MS lifetime and TO stellar mass (see Renzini and Buzzoni 1986). In particular, we have that

$$
\mathcal{B} \propto M_{T O}^{-s} \times \dot{M}_{T O}
$$

assuming that a power-law IMF holds with $d N_{*} \propto M_{*}^{-s} d M_{*}(s=2.35$ for the Salpeter case). Again, the r.h. term of eq. (10) is a direct output of stellar evolution theory, and $\mathcal{B}$ should therefore be regarded as a "universal" function, that is nearly insensitive to the specific input physics of the SSP models.

A comparison of the $\mathcal{B}$ scaling factor, as derived from the synthesis codes of Bruzual and Charlot (1993), Buzzoni (1989), and the Padova group (Bressan et al. 1994) for a SSP with $Z_{\odot}$ and Salpeter IMF is displayed in Fig. 4. While both the Buzzoni and Padova codes consistently match the fuel consumption theorem, the Bruzual and Charlot 


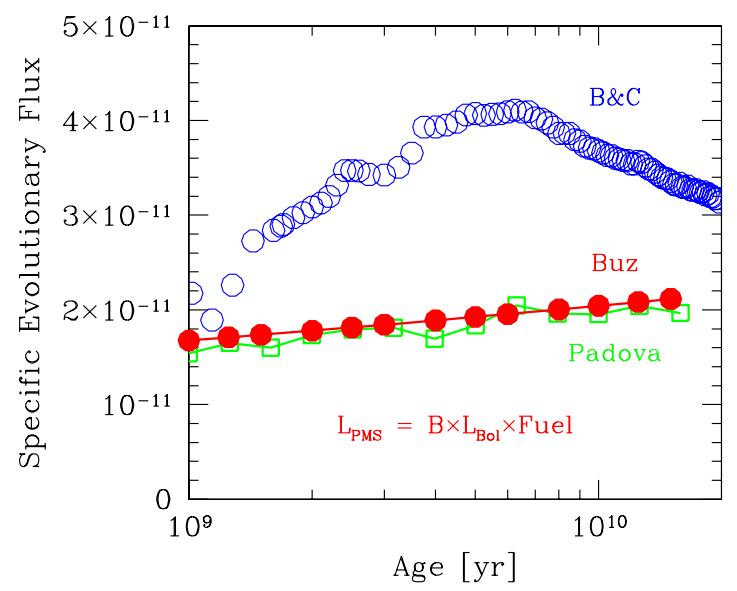

Figure 4. The "specific evolutionary flux", $\mathcal{B}$, for SSP models with $Z_{\odot}$ and Salpeter IMF according to Buzzoni (1989; "•" markers), Bressan et al. (1994; " $\square$ ") and Bruzual and Charlot (1993; "०"). The value of $\mathcal{B}$ is given in unit of $\mathrm{yr}^{-1} \mathrm{~L}_{\odot}^{-1}$. It directly scales Post-MS contribution to SSP luminosity through eq. (9).

models show a much higher value for the specific evolutionary flux. The effect is a macroscopic one, and reaches about a $100 \%$ discrepancy at intermediate age. Such higher scale factor leads, in eq. (9), to a corresponding overestimate of Post-MS luminosity at every age. The effect is quite shifty as it will not explicitly appear when considering luminosity evolution (cf. Fig. 5, left panel), but would strongly affect
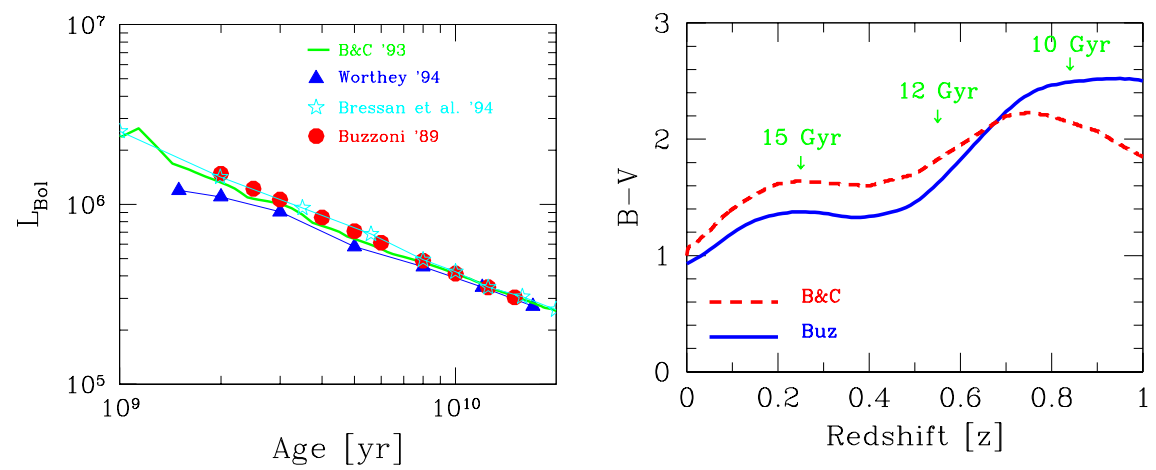

Figure 5. Left panel: bolometric evolution for a SSP with $Z_{\odot}$ and Salpeter IMF according to different synthesis codes, as labelled. Luminosity is normalized at 12 Gyr. Right panel: apparent $B-V$ evolution of elliptical galaxies according to Buzzoni (1995; "Buz") and Bruzual and Charlot (1993; "B\&C"). A cosmological model with $\left(H_{o}, q_{o}, z_{f}\right)=\left(50 \mathrm{~km} \mathrm{~s}^{-1} \mathrm{Mpc}^{-1}, 0,30\right)$ is assumed. The absolute age of galaxies at the different redshift is also labelled on the plot. 
the theoretical prediction of galaxy $\mathrm{M} / \mathrm{L}$ ratio and apparent color evolution. In the latter case, enhanced Post-MS contribution would lead to a larger number of red giant stars and therefore a systematically redder color for the stellar aggregate as a whole. This is shown in Fig. 5 (right panel) when comparing the Bruzual and Charlot (1993) results with the expected evolution of elliptical galaxies according to Buzzoni (1995).

\section{Acknowledgements}

It is a pleasure to thank the INAOE for the kind invitation and the excellent organization of this meeting. This work received partial financial support from the Italian MURST under COFIN '00 grant.

\section{References}

Bressan, A., Chiosi, C. \& Fagotto, F. Spectrophotometric Evolution of Elliptical Galaxies. I. Ultraviolet Excess and Color-magnitude-redshift Relations ApJS, 94:63-115, 1994.

Bruzual, G. \& Charlot, S. Spectral Evolution of Stellar Populations Using Isochrone Synthesis ApJ, 405:538-553, 1993.

Buzzoni, A. Evolutionary Population Synthesis in Stellar Systems. I - A Global Approach. ApJS, 71:817-869, 1989.

Buzzoni, A. Statistical Properties of Stellar Populations and Surface-Brightness Fluctuations in Galaxies. AESA, 275:433-450, 1993.

Buzzoni, A. Evolutionary Population Synthesis in Stellar Systems. II - Early-type Galaxies. ApJS, 98:69-101, 1995.

Buzzoni, A. Ultraviolet Properties of Primeval Galaxies: Theoretical Models from Stellar Population Synthesis. AJ, submitted, 2001.

Cohen, J.G., Hogg, D.W., Blandford, R., Cowie, L.L., Hu, E., Songaila, A., Shopbell, P. \& Richberg, K. Caltech Faint Galaxy Redshift Survey. X. A Redshift Survey in the Region of the Hubble Deep Field North ApJ, 538:29-52, 2000.

Fioc, M. \& Rocca-Volmerange, B. PEGASE: a UV to NIR Spectral Evolution Model of Galaxies $A \& A, 326: 950-962$.

Renzini, A. \& Buzzoni, A. Global Properties of Stellar Populations and the Spectral Evolution of Galaxies In A. Renzini and C. Chiosi, editors, Spectral evolution of galaxies, Dordrecht: Reidel, 1986, p. 195.

Tinsley, B. M. \& Gunn, J. E. Evolutionary Synthesis of the Stellar Population in Elliptical Galaxies. I - Ingredients, Broad-band Colors, and Infrared Features. ApJ, 203:52-62, 1976.

Tonry, J. \& Schneider, D.P. A new technique for measuring extragalactic distances. AJ, 96:807-815, 1988.

Worthey, G. Comprehensive Stellar Population Models and the Disentanglement of Age and Metallicity Effects ApJS, 95:107-149, 1994. 